\title{
Composição, riqueza e abundância de anuros em um remanescente de Cerrado e Mata Atlântica no estado de São Paulo
}

\author{
Cybele de Oliveira Araujo ${ }^{1,3}$ \& Selma Maria de Almeida-Santos ${ }^{2}$ \\ ${ }^{1}$ Seção de Animais Silvestres, Instituto Florestal - IF, Rua do Horto, 931, Horto Florestal, \\ CEP 02377-000, São Paulo, SP, Brasil \\ ${ }^{2}$ Laboratório de Ecologia e Evolução, Instituto Butantan, Av. Dr. Vital Brazil, 1500, \\ CEP 05503-900, São Paulo, SP, Brasil \\ ${ }^{3}$ Autor para correspondência: Cybele de Oliveira Araujo, e-mail: cyaraujo@if.sp.gov.br
}

ARAUJO, C.O. \& ALMEIDA-SANTOS, S.M. Composition, species richness and abundance of anurans in a Cerrado and Atlantic Forest remnant of the São Paulo state, Brazil. Biota Neotrop. 13(1): http://www. biotaneotropica.org.br/v13n1/en/abstract?inventory+bn02613012013

\begin{abstract}
The anurans of São Paulo state are the best known in the country. However, there are still considerable sampling gaps in the state, especially within natural vegetation remnants of the hydrographic basins of the Paranapanema river. The purpose of this study was to obtain information on species composition, species richness and abundance of anurans recorded at Estação Ecológica de Angatuba, a remnant of Cerrado and Atlantic Forest in the hydrographic basin of the Upper Paranapanema river. The survey was conducted in two phases: in March and November 2007, and between October 2008 and March 2009, totaling 38 sampling days. Surveys were conducted using pitfall traps, incidental encounters and acoustic and visual searches. We recorded 31 anuran species, belonging to 16 genera and eight families (Bufonidae, Centrolenidae, Craugastoridae, Cycloramphidae, Hylidae, Leiuperidae, Leptodactylidae and Microhylidae). Of this total, 15 species were new records to the Angatuba and Guareí municipalities. This research is an important contribution for expanding the knowledge of the anuran diversity in the Cerrado and Atlantic Forest, two priority global hotspots for biodiversity conservation in Brazil. Keywords: amphibians, Anura, diversity, inventory, Estação Ecológica de Angatuba.
\end{abstract}

ARAUJO, C.O. \& ALMEIDA-SANTOS, S.M. Composição, riqueza e abundância de anuros em um remanescente de Cerrado e Mata Atlântica no estado de São Paulo. Biota Neotrop. 13(1): http://www. biotaneotropica.org.br/v13n1/pt/abstract?inventory+bn02613012013

Resumo: Apesar da fauna de anuros no estado de São Paulo ser considerada a mais conhecida no país, ainda existem consideráveis lacunas amostrais no estado, sobretudo em remanescentes de vegetação natural das bacias hidrográficas do rio Paranapanema. Neste contexto, o objetivo deste estudo foi gerar informações sobre a composição, riqueza e abundância de anuros na Estação Ecológica de Angatuba, um remanescente de Cerrado e Mata Atlântica presente na bacia hidrográfica do Alto Paranapanema. O levantamento das espécies foi realizado em duas etapas de campo: março e novembro de 2007 e outubro de 2008 a março de 2009, totalizando 38 dias de inventário. A amostragem foi realizada por meio de armadilhas de interceptação e queda, encontro ocasional, procura auditiva e procura visual. Foram registradas 31 espécies de anuros pertencentes a 16 gêneros e oito famílias (Bufonidae, Centrolenidae, Craugastoridae, Cycloramphidae, Hylidae, Leiuperidae, Leptodactylidae e Microhylidae). Deste total, 15 espécies representam novos registros para os municípios de Angatuba e Guareí, SP. Este estudo é uma importante contribuição para ampliar o conhecimento sobre a diversidade de anuros no Cerrado e Mata Atlântica, os dois hotspots mundiais prioritários para a conservação biológica existentes no país. Palavras-chave: anfíbios, Anura, diversidade, inventário, Estação Ecológica de Angatuba. 


\section{Introdução}

Existem 230 espécies de anuros com ocorrência confirmada para o estado de São Paulo (Rossa-Feres et al. 2011), o que representa $25 \%$ da riqueza de espécies encontradas no país (Segalla et al. 2012). A anurofauna do estado pode ser dividida em dois grupos. Um deles é composto pelas espécies presentes nas fitofisionomias de floresta ombrófila da encosta litorânea e serras do Mar, Mantiqueira e Bocaina (Rossa-Feres et al. 2008), locais que ainda concentram a maior parte dos estudos sobre esta taxocenose no estado (Rossa-Feres et al. 2011). $\mathrm{O}$ outro grupo é formado por espécies associadas às formações de Cerrado e floresta estacional que ocorrem predominantemente no Planalto Ocidental Paulista (Rossa-Feres et al. 2008). Nos últimos anos houve um aumento significativo no número de trabalhos publicados sobre a diversidade de anuros em localidades de Cerrado (Ribeiro-Júnior \& Bertoluci 2009, Araujo \& Almeida-Santos 2011, Maffei et al. 2011b) e floresta estacional semidecidual (Araujo et al. 2009, Santos et al. 2009, Brassaloti et al. 2010, Maffei et al. 2011a) no interior do estado. Apesar da fauna de anuros no estado ser considerada como a mais conhecida do país, ainda existem lacunas de conhecimento no nordeste e sudeste do estado como na bacia hidrográfica do rio Paranapanema (Rossa-Feres et al. 2011).

Como resultado de processos históricos de uso e ocupação do solo, a Mata Atlântica tem sido reduzida pela supressão e fragmentação de sua cobertura vegetal, restando menos de $12 \%$ de sua extensão original de 1,3 milhões de $\mathrm{km}^{2}$, o que correspondia a 15\% do território brasileiro (Morellato \& Haddad 2000, Ribeiro et al. 2009). Estimativas recentes apontam que este domínio fitogeográfico apresenta-se sob a forma de fragmentos descontínuos, sendo a maior parte (80\%) menor que 50 ha (Ribeiro et al. 2009). Dentre as formações florestais brasileiras, a floresta estacional semidecidual é uma das mais ameaçadas e a que sofreu maior desmatamento, sobretudo em regiões onde a topografia propicia a agropecuária (Leitão-Filho 1987, Durigan et al. 2000, Ribeiro et al. 2009). Esta fitofisionomia apresenta-se permeada por formações de Cerrado, ocupando de forma bastante fragmentada parte dos estados do Paraná, São Paulo, Minas Gerais, Mato Grosso do Sul, Mato Grosso e Goiás e, em menor escala, Rio de janeiro, Espírito Santo e sul da Bahia (LeitãoFilho 1982). Alguns autores consideram que sua composição florística é uma transição entre a floresta ombrófila atlântica e o Cerrado, sendo classificada como um subconjunto da flora muito mais diversa da floresta ombrófila e provavelmente, composta por espécies capazes de sobreviver a uma estação seca mais prolongada (Leitão-Filho 1987, Oliveira-Filho \& Fontes 2000, Oliveira 2006). No entanto, estudos sugerem que esta formação vegetal seja considerada uma unidade fitogeográfica distinta, possuindo um número considerável de táxons endêmicos de plantas, lagartos e anuros (Prado 2000, Werneck \& Colli 2006, Iop et al. 2011, Werneck et al. 2011). Apesar da drástica redução em área, a Mata Atlântica é o domínio brasileiro que possui a maior riqueza de anuros apresentando mais de 400 espécies e pelo menos $85 \%$ de endemismo, desconsiderando-se várias espécies descritas mais recentemente (Cruz \& Feio 2007). Adicionalmente, 27 dos 39 modos reprodutivos de anuros conhecidos no mundo são encontrados neste domínio (Haddad \& Prado 2005).

O Cerrado, que possuía originalmente dois milhões de $\mathrm{km}^{2}$ de extensão (23\% do território nacional), compreende uma extensa área contínua nos estados de Goiás, Bahia, Minas Gerais e Mato Grosso e algumas penínsulas e áreas disjuntas que se estendem por outros estados como é o caso de São Paulo (Eiten 1972). No estado, o Cerrado apresenta-se sob a forma de manchas dispersas, especialmente na Depressão Periférica e no Planalto Ocidental Paulista (Durigan et al. 2004). Sua heterogeneidade ambiental, que inclui planaltos, depressões e planícies dominadas por formações campestres e savânicas e em menor proporção matas de galeria e floresta estacional (Oliveira-Filho \& Ratter 2002, Silva \& Bates 2002, Ab'Sáber 2005, Ribeiro \& Walter 2008), está refletida em sua biota reconhecida mundialmente como uma das mais ricas em espécies e endemismos e severamente ameaçada pelas atividades humanas (Colli et al. 2002, Mittermeier et al. 2004, Klink \& Machado 2005, Diniz-Filho et al. 2008). Foram registradas 209 espécies de anuros no Cerrado, das quais 51,7\% são endêmicas ao domínio (Valdujo et al. 2012). Assim como a Mata Atlântica, o Cerrado encontra-se bastante ameaçado, sendo a agricultura e a pecuária extensiva responsáveis pela maior parte da perda de áreas de vegetação natural (Ratter et al. 1997, Klink \& Machado 2005, Sano et al. 2010). Aproximadamente $40 \%$ da cobertura vegetal original do Cerrado foi devastada e a porção mais ao sul deste domínio é a mais severamente impactada, apresentando apenas 15\% de sua extensão original (Sano et al. 2010). No estado de São Paulo, somente 0,5\% deste domínio encontra-se protegido por unidades de conservação e os fragmentos remanescentes sofrem ameaças que incluem o isolamento, risco de incêndios e invasão por espécies vegetais exóticas (Durigan et al. 2004, 2007). É justamente nas regiões sul e sudoeste do Cerrado, que a riqueza de espécies de anuros tende a ser mais elevada (DinizFilho et al. 2008, Valdujo 2011).

Considerando que a fauna de anuros de uma porção significativa do país permanece pouco amostrada e estudos realizados no Cerrado e Mata Atlântica prevêem em um cenário de mudanças climáticas alterações na disponibilidade de áreas adequadas para ocorrência de muitas espécies de anuros, em especial as endêmicas e especialistas no uso do hábitat (Haddad et al. 2008a, Silvano 2011), torna-se imprescindível a realização de inventários e estudos padronizados de monitoramento dos anuros em localidades que se mantêm como lacunas amostrais. Os dados obtidos neste tipo de pesquisa contribuem de maneira inequívoca para uma avaliação mais adequada do status de conservação das espécies, declínios populacionais e extinções locais, informações estas indispensáveis para a elaboração de medidas efetivas de manejo e conservação das espécies (Verdade et al. 2012). Neste contexto, o objetivo deste estudo foi fornecer informações sobre a composição, riqueza, abundância e distribuição dos anuros na Estação Ecológica de Angatuba, um remanescente de Cerrado e floresta estacional semidecidual presente na bacia hidrográfica do Alto Paranapanema.

\section{Material e Métodos}

\section{Local de estudo}

A Estação Ecológica de Angatuba (23 24' S e 48 21' O; 625-750 m) localiza-se nos municípios de Angatuba e Guareí, bacia hidrográfica do Alto Paranapanema e apresenta área total de 1.394 ha (Monteiro et al. 2009) (Figura 1). O clima da região é do tipo Cwa de Köppen, com uma estação seca e outra chuvosa (Peel et al. 2007). A temperatura média nos meses mais quentes (janeiro e fevereiro) oscila ao redor de $24,6{ }^{\circ} \mathrm{C}$ e nos meses mais frios (junho e julho) entre 17 e $17,6{ }^{\circ} \mathrm{C}$ (Monteiro et al. 2009). A precipitação média anual é de aproximadamente $1.325 \mathrm{~mm}$, com a maior média de precipitação mensal ocorrendo no mês de janeiro $(195,5 \mathrm{~mm})$ e a menor em agosto (40 mm) (Monteiro et al. 2009). A cobertura vegetal desta estação ecológica é constituída predominantemente pela floresta estacional semidecidual montana $(56,9 \%$; 793,3 ha) e floresta estacional semidecidual aluvial (17,2\%; 239,8 ha), apresentando também algumas áreas de Cerrado $(17,7 \% ; 246,8$ ha $)$ em suas diversas fisionomias (cerradão, cerrado sentido restrito, campo sujo úmido) e uma pequena porção invadida por Pinus sp. (8,2\%; 114 ha) nos limites com a Floresta Estadual de Angatuba (Franco et al. 2009) (Figura 2). 


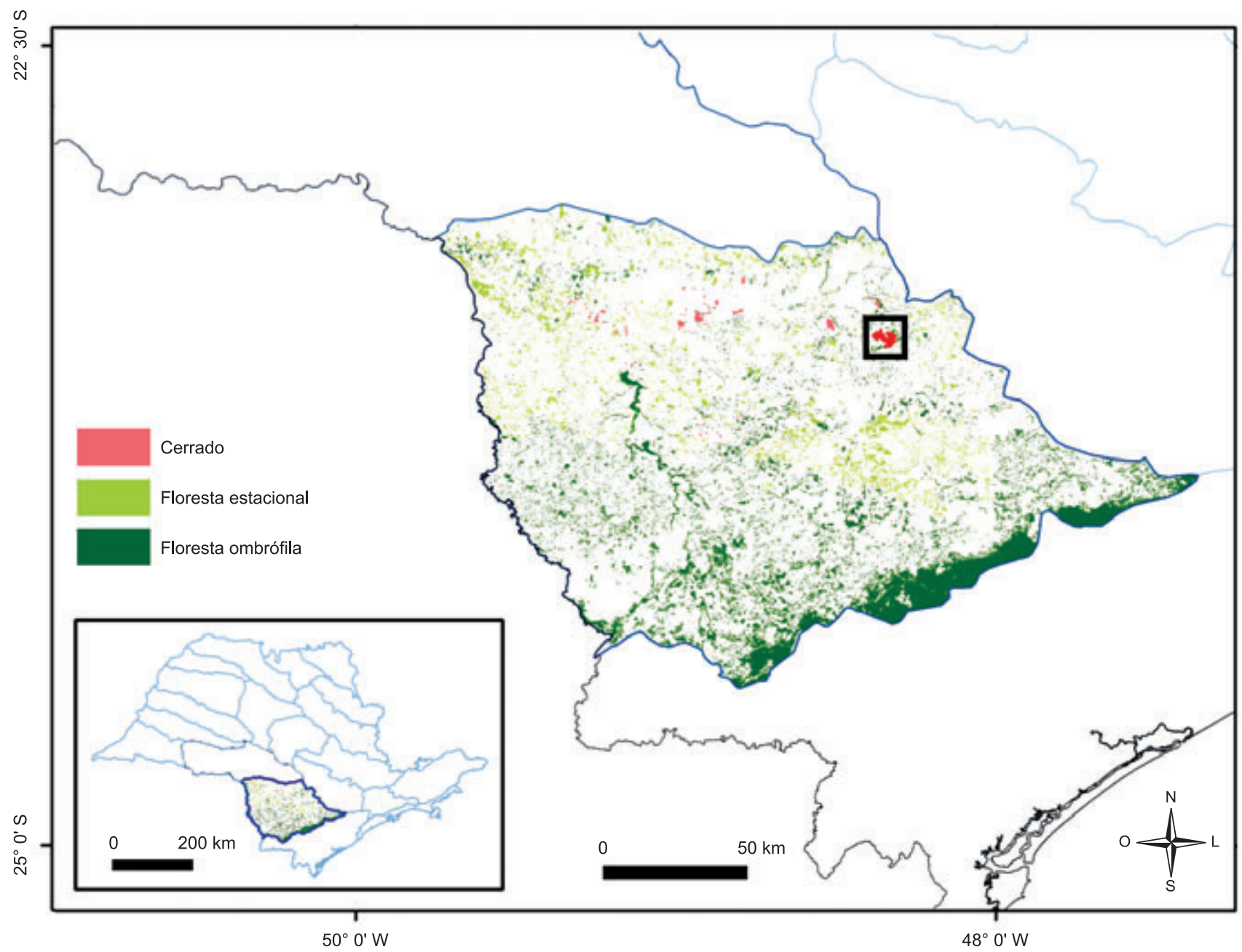

Figura 1. Remanescentes de vegetação natural da bacia hidrográfica do Alto Paranapanema, SP. O fragmento em vermelho representa a Estação Ecológica de Angatuba. No detalhe à esquerda, estão representadas as bacias hidrográficas do estado de São Paulo, com destaque para o Alto Paranapanema.

Figure 1. Natural vegetation remnants of the Upper Paranapanema river basin, São Paulo state, Brazil. The fragment in focus (in red) represents the Estação Ecológica de Angatuba. The detail on the left side depicts the river basins of São Paulo state, highlighting the Upper Paranapanema.

\section{Coleta e análise dos dados}

O inventário das espécies foi executado em dois períodos. A primeira fase foi de caráter exploratório, sendo realizada em duas etapas de campo que totalizaram oito dias de amostragem (março e novembro/2007). Já na segunda fase a amostragem foi efetuada em seis etapas de campo mensais, em um total de 30 dias de amostragem (outubro/2008 a março/2009).

Na primeira e segunda fase do levantamento de espécies foram utilizados os métodos de procura auditiva, procura visual e encontro ocasional (Crump \& Scott Junior 1994, Scott Junior \& Woodward 1994). A procura auditiva e visual consistiu em censos efetuados no período noturno, sendo examinados os microhábitats acessíveis (e.g. bromélias, folhiço, tronco de árvores) e os sítios reprodutivos utilizados pelos anuros (e.g. brejos, córregos, lagoas, várzeas). A duração da amostragem variou entre três e seis horas por noite, resultando em um esforço total de aproximadamente 100 horas de procura. Foram amostrados 14 ambientes aquáticos utilizados pelos anuros durante a reprodução (Figura 2, Apêndice 1). O método de encontro ocasional consistiu no registro de espécimes encontrados vivos ou mortos, quando não estava sendo executado nenhum tipo de procura. A utilização dos métodos de procura ativa visou apenas o registro das espécies, não sendo estimada a abundância dos indivíduos.

Adicionalmente, na segunda fase do inventário foram realizadas amostragens por meio de armadilhas de interceptação e queda (pitfall traps) interligadas por cercas-guia (drift-fences) (Corn 1994). Foram instaladas em diferentes fitofisionomias (cerrado denso, cerradão e floresta estacional semidecidual) 12 linhas compostas por quatro baldes plásticos de 60 litros, totalizando 48 armadilhas, com uma distância mínima de 500 m entre as linhas (Figura 2, Apêndice 1). Os baldes foram inseridos em buracos cavados no solo, sendo dispostos em linha reta (espaçados 15 metros entre si) e interligados por cercas-guia de aproximadamente $50 \mathrm{~cm}$ de altura. As armadilhas permaneceram abertas por cinco dias consecutivos (terceira ou última semana do mês), em um total de 30 dias de amostragem (esforço amostral $=1.440$ armadilhas/dia). Com o intuito de minimizar a predação dos anuros capturados nas armadilhas por vertebrados e invertebrados foram realizadas inspeções a cada 24 horas, sempre no período da manhã. Foram depositados abrigos em cada uma das armadilhas durante todo o período de amostragem para evitar a morte de indivíduos por dessecação e afogamento (Cechin \& Martins 2000). 


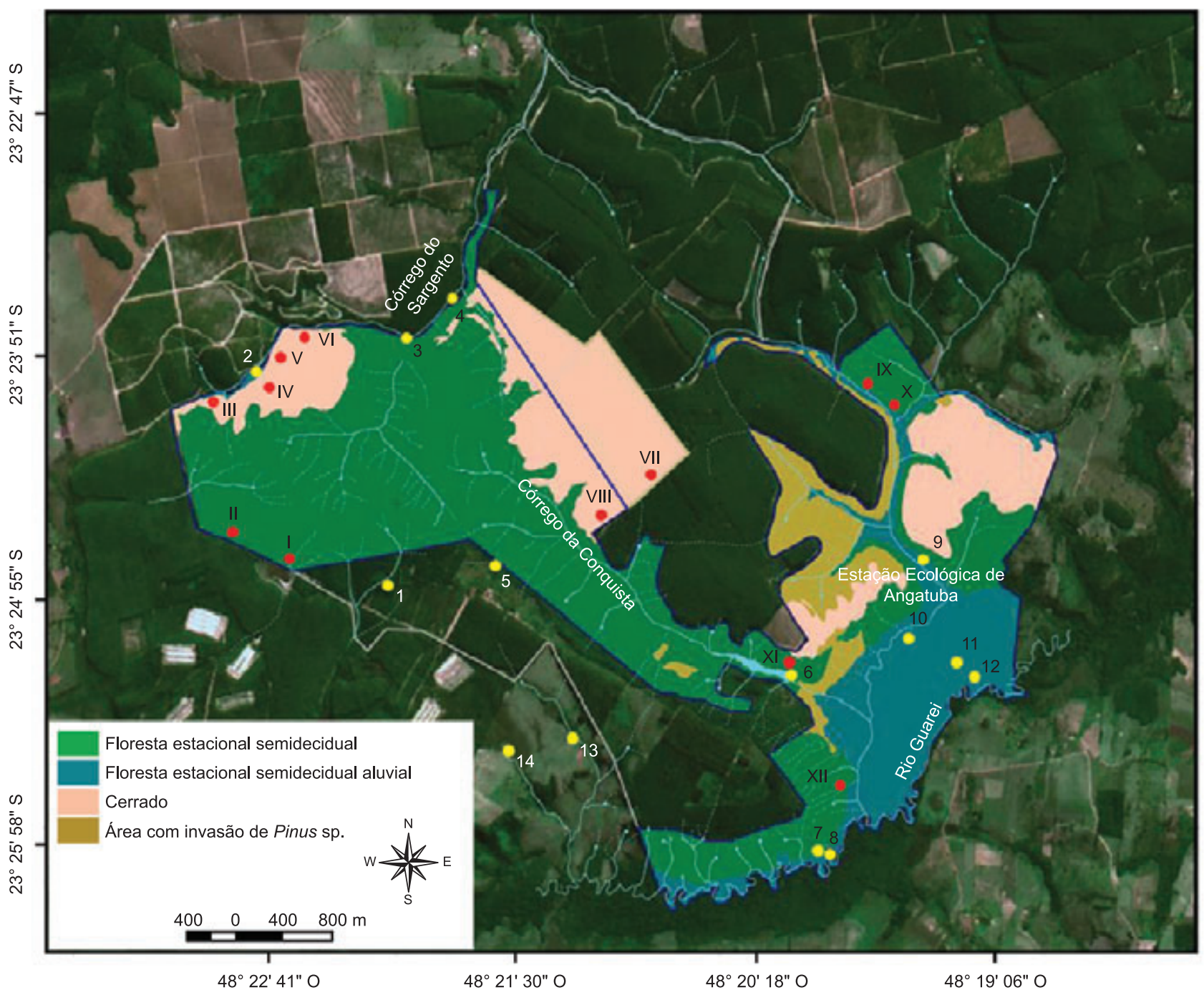

Figura 2. Cobertura vegetal da Estação Ecológica de Angatuba, SP e locais de amostragem (pontos em vermelho = linhas de armadilhas de interceptação e queda; pontos em amarelo = ambientes aquáticos). Fonte: modificado de Monteiro et al. (2009).

Figure 2. Vegetation of the Estação Ecológica de Angatuba, São Paulo state, Brazil and sampled areas (red dots = pitfall trap lines; yellow dots = aquatic sites). Source: modified from Monteiro et al. (2009).

Todos os espécimes capturados foram identificados, sendo coletados (máximo de 10 indivíduos por espécie) ou marcados pela amputação da extremidade de um dos artelhos e liberados próximos ao local de captura. Nas amostragens subsequentes, os indivíduos recapturados não foram considerados. Os espécimes testemunho (licenças de coleta IBAMA/RAN 10423-1 e 13706-2) foram depositados na Coleção Herpetológica do Museu de Zoologia, Universidade Estadual de Campinas (ZUEC) (Apêndice 2).

A eficiência da amostragem foi avaliada por meio de curvas de acumulação de espécies (Gotelli \& Colwell 2001) construídas a partir dos dados de incidência dos anuros registrados durante o período de inventário (uma amostra = um dia). Considerando os diversos estimadores de riqueza disponíveis, optou-se pela utilização do algoritmo Jackknife de primeira ordem (Magurran 2004, Walther \& Moore 2005, Hortal et al. 2006). As curvas foram geradas pelo programa EstimateS com 10.000 aleatorizações (Colwell 2009).

Para verificar se existiam registros de anuros para os municípios de Angatuba e Guareí antes da realização deste estudo, foram analisados os registros de tombo presentes nas principais coleções biológicas do estado de São Paulo: Coleção de Anfíbios do Departamento de Zoologia da Universidade Estadual Paulista "Júlio de Mesquita Filho”, campus de Rio Claro (CFBH), Coleção de Anfíbios da Universidade Estadual Paulista "Júlio de Mesquita Filho", campus de São José do Rio Preto (DZSJRP), Coleção Herpetológica do Museu de Zoologia da Universidade de São Paulo (MZUSP) e Coleção Herpetológica do Museu de Zoologia da Universidade Estadual de Campinas (ZUEC).

Apesar da existência de um sistema de classificação mais abrangente para os anuros (Pyron \& Wiens 2011), optou-se pela utilização da nomenclatura taxonômica mais consagrada apresentada por Frost (2013). O status de conservação das espécies foi obtido na lista vermelha internacional de espécies ameaçadas de extinção (International... 2012), livro vermelho da fauna brasileira ameaçada de extinção (Machado et al. 2008) e lista de anfíbios ameaçados do estado de São Paulo (Garcia et al. 2009). 


\section{Resultados e Discussão}

\section{Composição e distribuição das espécies}

Registraram-se na Estação Ecológica de Angatuba 31 espécies de anuros distribuídas em oito famílias: Bufonidae (2 espécies), Centrolenidae (1 espécie), Craugastoridae (1 espécie), Cycloramphidae (1 espécie), Hylidae (16 espécies), Leiuperidae (3 espécies), Leptodactylidae (5 espécies) e Microhylidae (2 espécies) (Figura 3, Tabela 1). Parte destas (29\%) pode ser considerada como generalista no uso do hábitat, sendo comumente encontradas em várias regiões do Brasil como Dendropsophus minutus, D. nanus, Hypsiboas albopunctatus, Leptodactylus fuscus, L. mystacinus, Physalaemus cuvieri, Rhinella schneideri, Scinax fuscomarginatus e S. fuscovarius (Haddad et al. 2008b, Frost 2013). No entanto, uma parcela das espécies amostradas (16\%) apresenta uma distribuição geográfica restrita a remanescentes de Mata Atlântica presentes em alguns estados do sul e sudeste do Brasil como Hypsiboas prasinus, Physalaemus olfersii, Rhinella ornata, Scinax rizibilis e Sphaenorhynchus caramaschii (Haddad et al. 2008b, Frost 2013) e outras estão fortemente associadas aos limites do Cerrado (10\%), apresentando uma grande dependência das formações vegetais abertas presentes neste domínio como Chiasmocleis albopunctata, Dendropsophus jimi e Eupemphix nattereri (Toledo et al. 2007, Valdujo 2011).

Espécies como Dendropsophus anceps, Haddadus binotatus, Hypsiboas prasinus, Physalaemus olfersii, Scinax rizibilis e Sphaenorhynchus caramaschii, encontradas comumente associadas à floresta ombrófila (Haddad et al. 2008b, Araujo et al. 2010, Forlani et al. 2010, Rossa-Feres et al. 2011), apresentam uma ocorrência ocasional

Tabela 1. Anuros da Estação Ecológica de Angatuba, SP. Fitofisionomias: $\mathrm{AA}=$ área alterada; $\mathrm{CD}=$ cerrado denso; $\mathrm{CE}=$ cerradão; $\mathrm{FES}=$ floresta estacional semidecidual; FESA = floresta estacional semidecidual aluvial. Métodos de amostragem: AIQ = armadilhas de interceptação e queda; PA = procura auditiva; $\mathrm{PV}=$ procura visual. Locais de captura: linhas de armadilhas e ambientes aquáticos.

Table 1. Anurans from Estação Ecológica de Angatuba, São Paulo state, Brazil. Phytophysiognomies: $A A=$ disturbed area; CD = cerrado denso; CE = cerradão; FES = seasonal semideciduous forest; FESA = seasonal semideciduous alluvial forest. Methods of sampling: AIQ = pitfall traps; PA = auditory encounters; PV = visual encounters. Sites of capture: pitfall trap lines and aquatic sites.

\begin{tabular}{|c|c|c|c|}
\hline Família/espécie & Fitofisionomia & Método & Linha de armadilha/ ambiente aquático \\
\hline \multicolumn{4}{|l|}{ Bufonidae } \\
\hline Rhinella ornata (Spix 1824) & CD; CE; FES; FESA & AIQ; PA/PV & III; VI; VII; VIII; IX; X; XI; XII; 9; 11; 12 \\
\hline Rhinella schneideri (Werner 1894) & AA; FES & $\mathrm{PA} / \mathrm{PV}$ & 5 \\
\hline \multicolumn{4}{|l|}{ Centrolenidae } \\
\hline Vitreorana uranoscopa (Müller 1924) & FES & $\mathrm{PA} / \mathrm{PV}$ & $2 ; 3$ \\
\hline \multicolumn{4}{|l|}{ Craugastoridae } \\
\hline Haddadus binotatus (Spix 1824) & CE; FES; FESA & AIQ & II; VII; X; XI; XII \\
\hline \multicolumn{4}{|l|}{ Cycloramphidae } \\
\hline $\begin{array}{l}\text { Odontophrynus americanus (Duméril \& Bibron 1841) } \\
\text { Hylidae }\end{array}$ & CE; FES; FESA & AIQ; PA/PV & III; 7 \\
\hline Aplastodiscus perviridis A. Lutz in B. Lutz 1950 & AA; CD; FES & $\mathrm{PA} / \mathrm{PV}$ & $1 ; 2 ; 3 ; 6 ; 13 ; 14$ \\
\hline Dendropsophus anceps (A. Lutz 1929) & FESA & $\mathrm{PA} / \mathrm{PV}$ & $8 ; 12$ \\
\hline Dendropsophus jimi (Napoli \& Caramaschi 1999) & AA & $\mathrm{PA} / \mathrm{PV}$ & $13 ; 14$ \\
\hline Dendropsophus minutus (Peters 1872) & AA; FES; FESA & $\mathrm{PA} / \mathrm{PV}$ & $1 ; 5 ; 6 ; 8 ; 12 ; 13 ; 14$ \\
\hline Dendropsophus nanus (Boulenger 1889) & AA; FES; FESA & $\mathrm{PA} / \mathrm{PV}$ & $1 ; 6 ; 7 ; 8 ; 12 ; 13$ \\
\hline Dendropsophus sanborni (Schmidt 1944) & AA; FES & $\mathrm{PA} / \mathrm{PV}$ & $9 ; 13 ; 14$ \\
\hline Hypsiboas albopunctatus (Spix 1824) & AA; FES; FESA & $\mathrm{PA} / \mathrm{PV}$ & $1 ; 5 ; 6 ; 7 ; 8 ; 9 ; 13 ; 14$ \\
\hline Hypsiboas faber (Wied-Neuwied 1821) & AA; FES; FESA & $\mathrm{PA} / \mathrm{PV}$ & $1 ; 6 ; 7 ; 12 ; 13 ; 14$ \\
\hline Hypsiboas prasinus (Burmeister 1856) & AA; FES; FESA & $\mathrm{PA} / \mathrm{PV}$ & $1 ; 4 ; 5 ; 8 ; 10 ; 11 ; 12 ; 13$ \\
\hline Itapotihyla langsdorffii (Duméril \& Bibron 1841) & FESA & $\mathrm{PA} / \mathrm{PV}$ & $8 ; 11$ \\
\hline Phyllomedusa tetraploidea Pombal \& Haddad 1992 & AA; FES; FESA & AIQ; PA/PV & I; 5; 7; 8; 9; 10; 13 \\
\hline Scinax fuscomarginatus (A. Lutz 1925) & AA & $\mathrm{PA} / \mathrm{PV}$ & $1 ; 6 ; 13 ; 14$ \\
\hline Scinax fuscovarius (A. Lutz 1925) & AA & $\mathrm{PA} / \mathrm{PV}$ & 1 \\
\hline Scinax rizibilis (Bokermann 1964) & FESA & $\mathrm{PA} / \mathrm{PV}$ & 7 \\
\hline Scinax squalirostris (A. Lutz 1925) & AA & $\mathrm{PA} / \mathrm{PV}$ & $13 ; 14$ \\
\hline Sphaenorhynchus caramaschii Toledo et al. 2007 & AA; FESA & $\mathrm{PA} / \mathrm{PV}$ & $8 ; 12 ; 14$ \\
\hline \multicolumn{4}{|l|}{ Leiuperidae } \\
\hline Eupemphix nattereri Steindachner 1863 & AA; CE; FES; & AIQ; PA/PV & 6; II; III; X; XII \\
\hline Physalaemus cuvieri Fitzinger 1826 & CD; CE; FES; FESA & AIQ; PA/PV & I; IV; VIII; X; XI; XII; 1; 5; 6; 8; 12; 13; 14 \\
\hline Physalaemus olfersii Lichtenstein \& Martens 1856 & FES; FESA & AIQ; PA/PV & IX; 13 \\
\hline \multicolumn{4}{|l|}{ Leptodactylidae } \\
\hline Leptodactylus fuscus (Schneider 1799) & AA; FESA & $\mathrm{PA} / \mathrm{PV}$ & $6 ; 8 ; 12 ; 13 ; 14$ \\
\hline Leptodactylus latrans (Steffen 1815) & AA & $\mathrm{PA} / \mathrm{PV}$ & 14 \\
\hline Leptodactylus mystacinus (Burmeister 1861) & AA; FES; FESA & AIQ; PA/PV & II; X; XII; 6; 12; 13; 14 \\
\hline Leptodactylus podicipinus (Cope 1862) & FES & AIQ & XI \\
\hline Leptodactylus sp. (gr. marmoratus) & FES; FESA & AIQ; PA/PV & XI; XII; 10 \\
\hline \multicolumn{4}{|l|}{ Microhylidae } \\
\hline Chiasmocleis albopunctata (Boettger 1885) & FES & AIQ & I; II \\
\hline Elachistocleis cesarii (Miranda-Ribeiro 1920) & $\mathrm{AA}$ & $\mathrm{PA} / \mathrm{PV}$ & $13 ; 14$ \\
\hline
\end{tabular}



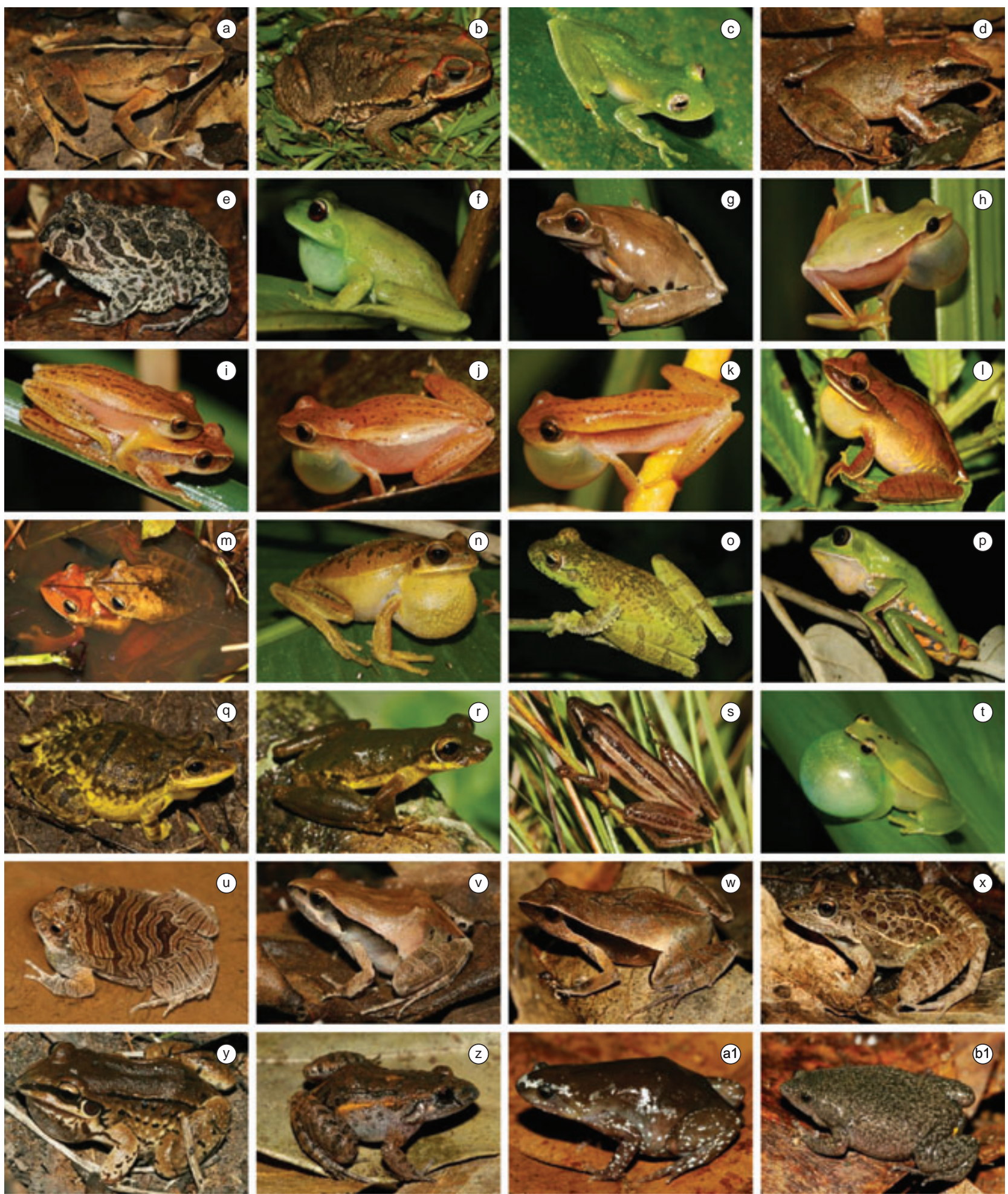

Figura 3. Espécies de anuros amostradas na Estação Ecológica de Angatuba, SP. a = Rhinella ornata; $\mathrm{b}=$ Rhinella schneideri; $\mathrm{c}=$ Vitreorana uranoscopa; $\mathrm{d}=$ Haddadus binotatus; $\mathrm{e}=$ Odontophrynus americanus; $\mathrm{f}=$ Aplastodiscus perviridis; $\mathrm{g}=$ Dendropsophus anceps; $\mathrm{h}=$ Dendropsophus jimi; $\mathrm{i}=$ Dendropsophus minutus; $\mathrm{j}=$ Dendropsophus nanus; $\mathrm{k}=$ Dendropsophus sanborni; $\mathrm{l}=$ Hypsiboas albopunctatus; $\mathrm{m}=$ Hypsiboas faber; $\mathrm{n}=$ Hypsiboas prasinus; $\mathrm{o}=$ Itapotihyla langsdorffi; $\mathrm{p}=$ Phyllomedusa tetraploidea $; \mathrm{q}=$ Scinax fuscovarius; $\mathrm{r}=$ Scinax rizibilis; $\mathrm{s}=$ Scinax squalirostris; $\mathrm{t}=$ Sphaenorhynchus caramaschii; $\mathrm{u}=$ Eupemphix nattereri; $\mathrm{v}$ = Physalaemus cuvieri; $\mathrm{w}$ = Physalaemus olfersii; $\mathrm{x}$ = Leptodactylus fuscus; $\mathrm{y}=$ Leptodactylus mystacinus; $\mathrm{z}=$ Leptodactylus sp. (gr. marmoratus); a1 = Chiasmocleis albopunctata; b1 = Elachistocleis cesarii. Todos os indivíduos são da localidade estudada (fotos: Cybele O. Araujo).

Figure 3. Anuran species sampled at the Estação Ecológica de Angatuba, São Paulo state, Brazil. a = Rhinella ornata; $\mathrm{b}=$ Rhinella schneideri; $\mathrm{c}=$ Vitreorana uranoscopa; $\mathrm{d}$ = Haddadus binotatus; $\mathrm{e}=$ Odontophrynus americanus; $\mathrm{f}=$ Aplastodiscus perviridis; $\mathrm{g}$ = Dendropsophus anceps; $\mathrm{h}=$ Dendropsophus jimi; $\mathrm{i}$ = Dendropsophus minutus; $\mathrm{j}=$ Dendropsophus nanus; $\mathrm{k}=$ Dendropsophus sanborni; $\mathrm{l}$ = Hypsiboas albopunctatus; $\mathrm{m}$ = Hypsiboas faber; $\mathrm{n}=$ Hypsiboas prasinus; $\mathrm{o}=$ Itapotihyla langsdorffii; $\mathrm{p}=$ Phyllomedusa tetraploidea $; \mathrm{q}=$ Scinax fuscovarius; $\mathrm{r}=$ Scinax rizibilis; $\mathrm{s}=$ Scinax squalirostris; $\mathrm{t}=$ Sphaenorhynchus caramaschii; $\mathrm{u}=$ Eupemphix nattereri; $\mathrm{v}=$ Physalaemus cuvieri; $\mathrm{w}=$ Physalaemus olfersii; $\mathrm{x}=$ Leptodactylus fuscus $; \mathrm{y}=$ Leptodactylus mystacinus; $\mathrm{z}=$ Leptodactylus sp. (gr. marmoratus); a1 = Chiasmocleis albopunctata; b1 = Elachistocleis cesarii. All individuals were found at the study site (photos: Cybele O. Araujo). 
na floresta estacional semidecidual. A presença destes anuros na Estação Ecológica de Angatuba pode ser reflexo da transição existente entre estas duas fitofisionomias no Alto Paranapanema, sobretudo na porção sul e sudeste desta bacia hidrográfica (Kronka et al. 2005). Das espécies registradas nesta localidade, 15 espécies representam novos registros para os municípios de Angatuba e Guareí: Dendropsophus anceps, D. minutus, D. nanus, D. sanborni, Hypsiboas faber, $H$. prasinus, Itapotihyla langsdorffii, Leptodactylus podicipinus, L. sp. (gr. marmoratus), Rhinella schneideri, Scinax fuscomarginatus, S. rizibilis, S. squalirostris, Sphaenorhynchus caramaschii e Vitreorana uranoscopa. A captura de Scinax squalirostris no município de Angatuba representou o primeiro registro da espécie para a bacia hidrográfica do Alto Paranapanema e existe a possibilidade de Leptodactylus sp. (gr. marmoratus) representar uma espécie ainda não descrita (B. V. M. Berneck; A. Fouquet, comunicação pessoal). Apesar de nenhuma das espécies estudadas estar inserida nas listas de anfíbios ameaçados de extinção (International... 2012, Machado et al. 2008, Garcia et al. 2009), possivelmente, as populações de anuros que ocorrem associadas as formações de Cerrado representam o limite de distribuição mais austral das espécies neste domínio fitogeográfico (Diniz-Filho et al. 2008, Valdujo 2011) e apenas a conservação e conexão destes remanescentes de vegetação podem assegurar a manutenção da diversidade genética destas espécies.

As espécies Chiasmocleis albopunctata, Dendropsophus anceps, Itapotihyla langsdorffii, Leptodactylus podicipinus, L. sp. (gr. marmoratus), Physalaemus olfersii, Scinax rizibilis e Vitreorana uranoscopa foram registradas apenas em áreas com cobertura vegetal de floresta estacional semidecidual, sendo que Chiasmocleis albopunctata e Leptodactylus podicipinus não foram observadas em atividade de vocalização como os demais anuros citados (Tabela 1). Nenhuma das espécies foi encontrada exclusivamente nas fitofisionomias de Cerrado (cerrado denso e cerradão). Algumas das espécies amostradas neste estudo e associadas a ambientes aquáticos em formações abertas de Cerrado durante o período reprodutivo foram verificadas somente em áreas alteradas no entorno dessa estação ecológica como Dendropsophus jimi, Elachistocleis cesarii, Leptodactylus latrans, Scinax fuscomarginatus, S. fuscovarius e S. squalirostris (Tabela 1). Já os anuros Aplastodiscus perviridis,
Eupemphix nattereri, Haddadus binotatus, Odontophrynus americanus, Physalaemus cuvieri e Rhinella ornata foram registradas tanto nas formações de Cerrado como na floresta estacional (Tabela 1), sendo que apenas Haddadus binotatus não foi avistada vocalizando. Poucas espécies (21\%) foram observadas em atividade de vocalização restrita aos corpos d’água lóticos (e.g. rios, córregos) como Odontophrynus americanus, Physalaemus olfersii, Rhinella schneideri, Scinax rizibilis e Vitreorana uranoscopa, sendo a maior parte (39\%) encontrada vocalizando em corpos d'água lênticos (e.g. lagoas, brejos, várzeas) ou em ambos os tipos de ambiente aquático (39\%) (Tabela 1, Apêndice 1). Padrão semelhante no uso do hábitat foi verificado em outras localidades no estado que apresentam ecótono entre formações de Cerrado e floresta estacional semidecidual (Vasconcelos \& Rossa-Feres 2005, Araujo et al. 2009, Araujo \& Almeida-Santos 2011, Maffei et al. 2011a).

A composição de anuros observada é resultado da posição geográfica da localidade estudada, apresentando espécies associadas ao Cerrado e as fitofisionomias de Mata Atlântica, como a floresta estacional e floresta ombrófila. Estudos apontaram uma grande similaridade faunística entre as taxocenoses de anuros que ocorrem em áreas de Cerrado e floresta estacional semidecidual no estado de São Paulo (Araujo et al. 2009, Santos et al. 2009). Esta similaridade pode ser atribuída ao fato da floresta estacional semidecidual no estado possuir características de descontinuidade, apresentandose permeada por manchas dispersas de Cerrado em suas diversas formações (Leitão-Filho 1987, Durigan et al. 2004). Já a presença de algumas espécies típicas de floresta ombrófila nesta localidade é reflexo da existência de uma transição entre esta fitofisionomia e a floresta estacional no Alto Paranapanema.

\section{Riqueza e abundância das espécies}

As curvas de acumulação de espécies para 21 dias de amostragem por procura auditiva e visual e 30 dias por armadilhas de interceptação e queda, apesar de não atingirem uma assíntota, apresentaram tendência à estabilização (Figura 4), indicando que, possivelmente, a maior parte das espécies foi registrada durante o estudo. $\mathrm{O}$ estimador de riqueza Jackknife I não indicou diferenças entre as riquezas de anuros estimadas e observadas obtidas pelos diferentes
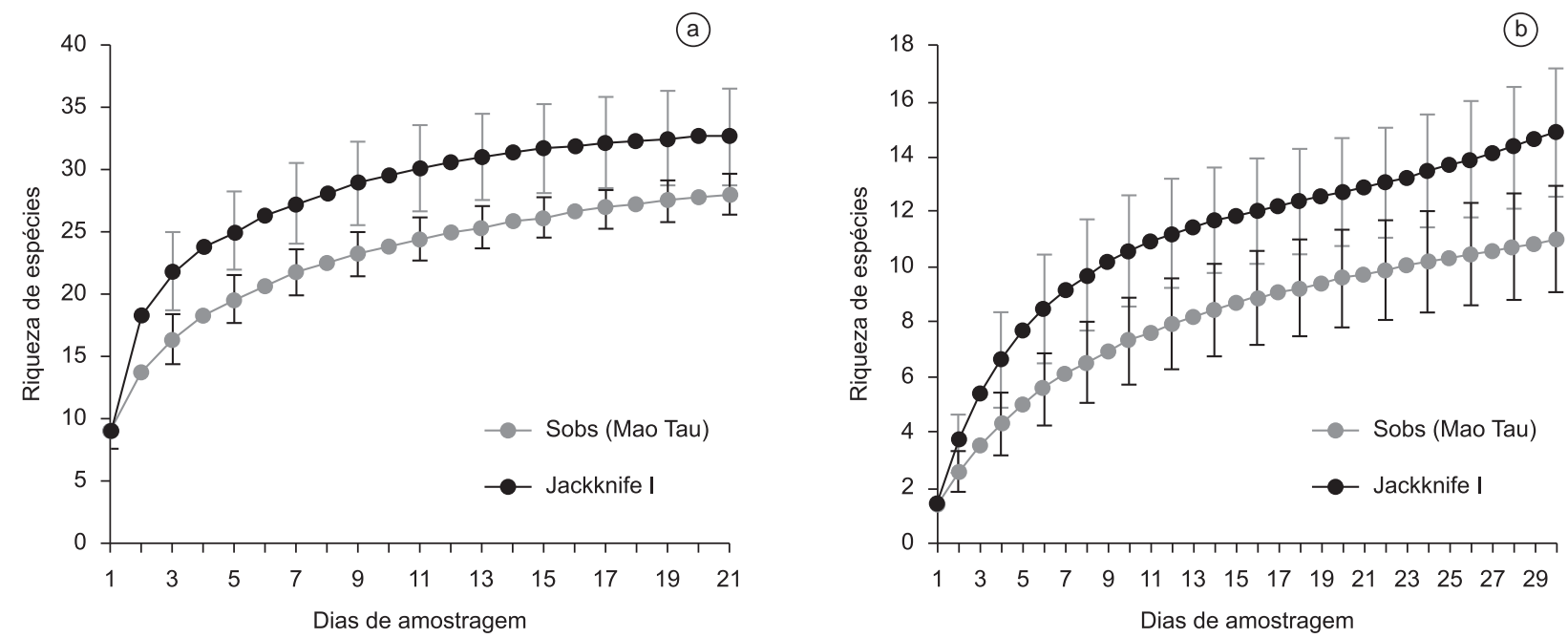

Figura 4. Curvas de acumulação de espécies representando a riqueza observada (linha preta) e estimada pelo estimador Jackknife I (linha cinza) para 21 dias de amostragem por procura auditiva e visual (a) e 30 dias por armadilhas de interceptação e queda (b) na Estação Ecológica de Angatuba, SP. As barras verticais correspondem aos desvios padrão da riqueza de espécies observada e estimada.

Figure 4. Species accumulation curves representing the species richness observed (black line) and estimated by Jackknife I estimator (gray line) for 21 sampling days using acoustic and visual searches (a) and 30 days using pitfall traps (b) at the Estação Ecológica de Angatuba, São Paulo state, Brazil. Vertical bars represent the standard deviations of species richness observed and estimated. 


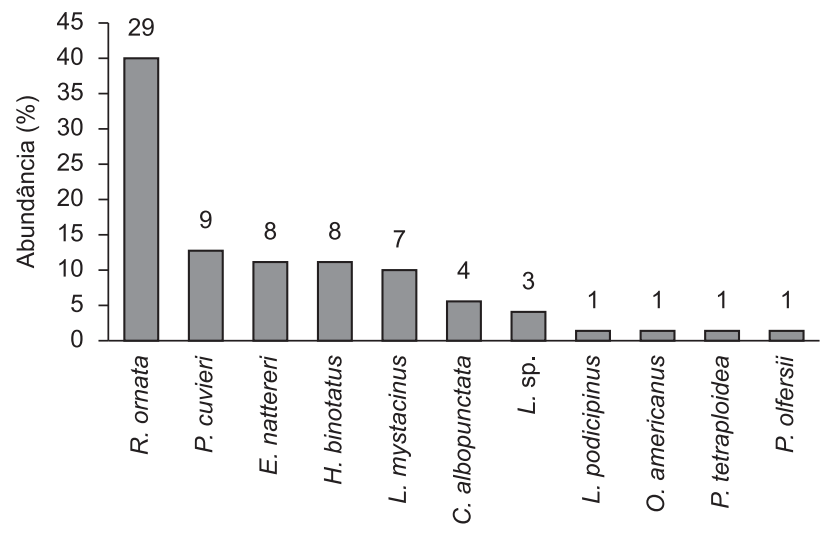

Figura 5. Abundância relativa das espécies de anuros amostradas por meio de armadilhas de interceptação e queda na Estação Ecológica de Angatuba, SP. Os valores sobre as barras representam o número de indivíduos registrados para cada espécie (número total de indivíduos $=72$ ).

Figure 5. Relative abundance of anuran species sampled by pitfall traps at the Estação Ecológica de Angatuba, São Paulo state, Brazil. The values on the bars represent the number of individuals recorded for each species (total number of individuals $=72$ ).

métodos de amostragem. Grande parte dos espécimes registrados (28 espécies, 90\%) foi amostrada por meio da procura auditiva e visual nos ambientes aquáticos utilizados pelos anuros durante o período reprodutivo, sendo a maioria composta por representantes da família Hylidae (16 espécies, 57\%) (Tabela 1). Nas armadilhas de interceptação e queda foram capturadas apenas 11 espécies (35\%) (Figura 5, Tabela 1), no entanto este método permitiu a captura de Chiasmocleis albopunctata, Haddadus binotatus e Leptodactylus podicipinus que não foram detectadas pela busca ativa. Apesar de alguns autores apontarem que os estimadores não-paramétricos apresentam uma grande dependência da riqueza de espécies observada e, portanto, do número e tamanho das amostras (Melo \& Froehlich 2001, Melo 2004), ainda sim a riqueza estimada neste inventário pode fornecer uma base para comparações com outros levantamentos realizados em localidades de Cerrado e floresta estacional semidecidual que utilizaram este mesmo estimador de riqueza (e.g. Ribeiro-Júnior \& Bertoluci 2009, Santos et al. 2009, Brassaloti et al. 2010, Araujo \& Almeida-Santos 2011, Valdujo et al. 2011).

Inventários de anuros realizados em unidades de conservação de Cerrado (Estação Ecológica de Santa Bárbara; Araujo 2012) e floresta estacional semidecidual (Estação Ecológica de Bauru; dados não publicados) obtiveram a mesma riqueza de espécies em armadilhas de interceptação e queda (baldes de 60 litros) com esforços amostrais similares ao empregado neste estudo (1.440 e 1.320 armadilhas/dia, respectivamente). As armadilhas de interceptação e queda são mais apropriadas para o registro de espécies de hábitos terrestres como anuros das famílias Bufonidae, Craugastoridae, Cycloramphidae, Leiuperidae, Leptodactylidae e Microhylidae, especialmente as espécies subterrâneas e criptozóicas, que são mais difíceis de serem detectadas pelos métodos tradicionais de procura visual (Cechin \& Martins 2000). Considerando os anuros inventariados pertencentes a estas famílias, quatro espécies não foram capturadas por este método (Elachistocleis cesarii, Leptodactylus fuscus, L. latrans e Rhinella schneideri) (Tabela 1). Foram coletados nas armadilhas apenas 72 indivíduos, sendo Rhinella ornata a espécie mais abundante (29 indivíduos; 40,3\%) e observada em todas fitofisionomias analisadas (cerrado denso, cerradão, floresta estacional semidecidual e floresta estacional semidecidual aluvial) (Figura 5).

Estudo comparando a efetividade dos métodos de procura auditiva e visual e de armadilhas de interceptação e queda na amostragem de anuros em área de floresta estacional semidecidual (Parque Estadual do Morro do Diabo; Vasconcelos et al. 2010) obteve resultados semelhantes aos encontrados no presente estudo, demonstrando uma baixa eficiência das armadilhas de interceptação e queda na captura dos espécimes. Apesar da menor eficiência da amostragem passiva, é importante considerar que existem limitações inerentes a cada método e apenas a utilização de métodos complementares (amostragem ativa e passiva) propicia a obtenção de listas de espécies mais completas (Silveira et al. 2010). Embora os custos financeiros e o esforço empregado na instalação e manutenção das armadilhas de interceptação e queda sejam mais elevados que os necessários para a realização da procura auditiva e visual (Corn 1994, Cechin \& Martins 2000) é desejável que a amostragem passiva seja empregada em inventários da herpetofauna. Isto por que, além de permitir a amostragem simultânea de diversos locais, este método permite a obtenção de dados padronizados de riqueza e abundância das espécies, o que favorece comparações mais confiáveis entre diferentes localidades (Silveira et al. 2010).

A riqueza de espécies observada na Estação Ecológica de Angatuba (31 espécies) mostrou-se superior às registradas em outras unidades de conservação do estado que também apresentam ecótono entre Cerrado e floresta estacional semidecidual como a Estações Ecológicas de Jataí (27 espécies; D. T. Corrêa, comunicação pessoal) e o Parque Estadual das Furnas do Bom Jesus (24 espécies; Araujo et al. 2009). Esta superioridade se deve a presença de algumas espécies típicas da floresta ombrófila na taxocenose estudada, o que não ocorre nas outras localidades citadas. Já o número de indivíduos capturados pode ser considerado baixo em relação aos obtidos em inventários efetuados em outras localidades do estado com estas formações vegetais e esforços amostrais equivalentes como nas estações ecológicas de Assis (141 indivíduos; Araujo \& Almeida-Santos 2011), Bauru (483 indivíduos; C. O. Araujo, dados não publicados) e Santa Bárbara (331 indivíduos; Araujo 2012). Esta baixa abundância pode estar relacionada ao fato de apenas uma das seis campanhas de campo (mês de janeiro) ter ocorrido em dias chuvosos. Sabe-se que em regiões tropicais, com a sazonalidade bem marcada, a ocorrência e a reprodução de grande parte dos anuros estão associadas às estações do ano que apresentam maior pluviosidade (primavera e verão), sendo o encontro dos indivíduos favorecido pela ocorrência de chuva (Haddad et al. 2008b, Uetanabaro et al. 2008, Silveira et al. 2010).

\section{Agradecimentos}

Somos gratas à responsável técnica (Bárbara H. S. Prado) pelo apoio logístico e aos funcionários da Estação Ecológica de Angatuba (Benedito C. Oliveira, João J. Marques, João B. A. Santos e Valmir Machado) pela grande ajuda durante os trabalhos de campo; à Thais $\mathrm{H}$. Condez e Décio T. Corrêa Filho pelo auxílio nas etapas iniciais deste estudo; ao Célio F. B. Haddad pela contribuição na identificação dos espécimes e ao Instituto Florestal pelo suporte financeiro. Selma M. Almeida-Santos agradece ao CNPq e a FAPESP pelo apoio financeiro.

\section{Referências Bibliográficas}

AB’SÁBER, A.N. 2005. Os domínios de natureza no Brasil: potencialidades paisagísticas. Ateliê Editorial, São Paulo.

ARAUJO, C.O. \& ALMEIDA-SANTOS, S.M. 2011. Herpetofauna de um remanescente de Cerrado no estado de São Paulo, sudeste do Brasil. Biota Neotrop. 11(3): http://www.biotaneotropica.org.br/v11n3/pt/ abstract?article+bn00511032011 (último acesso em 06/06/2012).

ARAUJO, C.O. 2012. Composição, riqueza e abundância de anfíbios e répteis no Alto e Médio Paranapanema, estado de São Paulo. Tese de Doutorado, Universidade de São Paulo, São Paulo. 
ARAUJO, C.O., CONDEZ, T.H. \& SAWAYA, R.J. 2009. Anfíbios anuros do Parque Estadual das Furnas do Bom Jesus, sudeste do Brasil, e suas relações com outras taxocenoses no Brasil. Biota Neotrop. 9(2): http:// www.biotaneotropica.org.br/v9n2/pt/abstract?article+bn01109022009 (último acesso em 13/02/2010).

ARAUJO, C.O., CONDEZ, T.H., BOVO, R.P., CENTENO, F.C. \& LUIZ, A.M. 2010. Amphibians and reptiles of the Parque Estadual Turístico do Alto Ribeira (PETAR), SP: an Atlantic Forest remnant of southeastern Brazil. Biota Neotrop. 10(4): http://www.biotaneotropica.org.br/v10n4/ pt/abstract?inventory+bn01710042010 (último acesso em 02/03/2011).

BRASSALOTI, R.A., ROSSA-FERES, D.C. \& BERTOLUCI, J. 2010. Anurofauna da Floresta Estacional Semidecidual da Estação Ecológica dos Caetetus, sudeste do Brasil. Biota Neotrop. 10(1): http://www. biotaneotropica.org.br/v10n1/pt/abstract?inventory+bn01810012010 (último acesso em 16/07/2010).

CECHIN, S.Z. \& MARTINS, M. 2000. Eficiência de armadilhas de queda (Pitfall traps) em amostragens de anfíbios e répteis no Brasil. Rev. Bras. Zool. 17(3): 729-749. http://dx.doi.org/10.1590/S010181752000000300017

COLLI, G.R., BASTOS, R.P. \& ARAÚJO, A.F.B. 2002. The character and dynamics of the Cerrado herpetofauna. In The cerrados of Brazil ecology and natural history of a Neotropical Savanna (P.S. Oliveira \& R.J. Marquis, eds.). Columbia University Press, New York, p.223-241.

COLWELL, R.K. 2009. EstimateS: statistical estimation of species richness and shared species from samples. versão 8. 2. 0. http://viceroy.eeb.uconn. edu/estimates (último acesso em: 10/07/2010).

CORN, P.S. 1994. Straight-line drift-fences and pitfall traps. In Measuring and monitoring biological diversity. Standard methods for amphibians (W.R. Heyer, M.A. Donnely, R.W. McDiarmid, L.C. Hayek \& M.S. Foster, eds.). Smithsonian institution Press, Washington and London, p.109-117.

CRUMP, M.L. \& SCOTT JUNIOR, N.J. 1994. Visual encounter surveys. In Measuring and monitoring biological diversity: standard methods for amphibians (W.R. Heyer, M.A. Donnelly, R.W. Mc Diarmid, L.C. Hayek \& M.S Foster, eds.). Smithsonian Institution Press. Washington and London, p.84-92.

CRUZ, C.A.G. \& FEIO, R.N. 2007. Endemismos em anfíbios em áreas de altitude na Mata Atlântica no sudeste do Brasil. In Herpetologia no Brasil II (L.B. Nascimento \& M.E. Oliveira, eds.). Sociedade Brasileira de Herpetologia, Belo Horizonte, p.117-126.

DINIZ-FILHO, J.A.F., BINI, L.M., VIEIRA, C.M., BLAMIRES, D. TERRIBILE, L., BASTOS, R., OLIVEIRA, G. \& BARRETO, B. 2008. Spatial patterns of terrestrial vertebrate species richness in the Brazilian Cerrado. Zool. Stud. 47(2): 146-157.

DURIGAN, G., FRANCO, G.A.D.C. \& SIQUEIRA, M.F. 2004. A vegetação dos remanescentes de cerrado no Estado de São Paulo. In Viabilidade da conservação dos remanescentes de Cerrado no Estado de São Paulo (M.D. Bittencourt \& R.R. Mendonça, orgs.). Annablume, FAPESP, São Paulo, p.29-56.

DURIGAN, G., FRANCO, G.A.D.C., SAITO, M. \& BAITELO, J.B. 2000 Estrutura e diversidade do componente arbóreo da floresta na Estação Ecológica dos Caetetus, Gália, SP. Rev. bras. Bot. 23(4):371-383.

DURIGAN, G., SIQUEIRA, M.F. \& FRANCO, G.A.D.C. 2007. Threats to the Cerrado remnants of the State of São Paulo, Brazil. Braz. Sci. Agric. 64(4):355-363.

EITEN, G. 1972. The cerrado vegetation of Brazil. Bot. Rev. 38:201-341. http://dx.doi.org/10.1007/BF02859158

FORLANI, M.C., BERNARDO, P.H., HADDAD, C.F.B. \& ZAHER, H. 2010 Herpetofauna of the Carlos Botelho State Park, São Paulo state, Brazil. Biota Neotrop. 10(3): http://www.biotaneotropica.org.br/v10n3/en/abst ract?inventory+bn00210032010.

FRANCO, G.A.D.C., SOUZA, F.M., ESTEVES, R., DURIGAN, G., PASTORE, J.A., BAITELLO, J.B., TONIATO, M.T.Z., KANASHIRO, M.M., IVANAUSKAS, N.M. \& AGUIAR, O.T. 2009. Vegetação. In Plano de manejo da Estação Ecológica de Angatuba (C.H.B. Monteiro, B.H.S. Prado \& A.C. Dias, orgs.). Instituto Florestal/SEMA, São Paulo, p.107-117.

FROST, D.R. 2013. Amphibian species of the world: an online reference. Version 5.6. Electronic Database. American Museum of Natural History, New York, USA. http://research.amnh.org/herpetology/amphibia/index. html (último acesso em 06/02/2013).
GARCIA, P.C.A., SAWAYA, R.J., MARTINS, I.A., BRASILEIRO, C.A., VERDADE, V.K., JIM, J., SEGALLA, M.V., MARTINS, M., ROSSAFERES, D.C., HADDAD, C.F.B., TOLEDO, L.F., PRADO, C.P.A., BERNECK, B.M. \& ARAÚJO, O.G.S. 2009. Anfíbios. In Fauna ameaçada de extinção no estado de São Paulo: vertebrados (M. Bressan, m.C.m. Kierulff \& a.m. Sugieda, orgs.). Fundação Parque Zoológico de São Paulo/SEMA, São Paulo, p.329-347.

GOTELLI, N.J. \& COWELL, R.K. 2001. Quantifying biodiversity: procedures and pitfalls in the measurement and comparison of species richness. Ecol. Lett. 4(4):379-391. http://dx.doi.org/10.1046/j.1461-0248.2001.00230

HADDAD, C.F.B. \& PRADO, C.P.A. 2005. Reproductive modes in frogs and their unexpected diversity in the Atlantic Forest of Brazil. BioScience 55(3):207-217. http://dx.doi.org/10.1641/00063568(2005)055[0207:RMIFAT]2.0.CO;2

HADDAD, C.F.B., GIOVANELLI, J.G.R. \& ALEXANDRINO, J. 2008a. O aquecimento global e seus efeitos na distribuição e declínios dos anfíbios. In Biologia e mudanças climáticas no Brasil (M.S. Buckeridge, org.). RiMa, São Carlos, p.195-206.

HADDAD, C.F.B., TOLEDO, L.F. \& PRADO, C.P.A. 2008b. Anfíbios da Mata Atlântica: guia dos anfíbios anuros da Mata Atlântica. Neotropica, São Paulo.

HORTAL, J., BORGES, P.A.V. \& GASPARE, C. 2006. Evaluating the performance of species richness estimators: sensitivity to sample grain size. J. Anim. Ecol. 75(1):274-287. http://dx.doi.org/10.1111/j.13652656.2006.01048.x

INTERNATIONAL UNION FOR CONSERVATION OF NATURE - IUCN. 2012. The IUCN red list of threatened species. Version 2012.2. http://www.iucnredlist.org (último acesso em: 26/02/2013).

IOP, S., CALDART, V.M., SANTOS, T.G. \& Cechin, S.Z. 2011. Anurans of Turvo State Park: testing the validity of Seasonal Forest as a new biome in Brazil. Nat. Hist. 45(39-40):2443-2461. http://dx.doi.org/10.1080/00 222933.2011.596951

KLINK, C.A. \& MACHADO, R.B. 2005. Conservation of the Brazilian Cerrado. Conserv. Biol. 19(3):707-713. http://dx.doi.org/10.1111/j.15231739.2005.00702.x

KRONKA, F.J.N., NALON, M.A. \& MATSUKUMA, C.K. 2005. Inventário florestal da vegetação natural do estado de São Paulo. Instituto Florestal/ SEMA, São Paulo.

LEITÃO-FILHO, H.F. 1982. Aspectos taxonômicos das florestas do estado de São Paulo. Silvicultura em São Paulo. 16A(1):197-206.

LEITÃO-FILHO, H.F. 1987. Considerações sobre a florística de florestas tropicais e sub-tropicais do Brasil. I. P. E. F. 35:41-46.

MACHADO, A.B.M., DRUMMOND, G.M. \& PAGLIA, A.P. 2008. Livro vermelho da fauna brasileira ameaçada de extinção. Fundação Biodiversitas/MMA, Belo Horizonte.

MAFFEI, F., UBAID, F.K. \& JIM, J. 2011a. Anfíbios: Fazenda Rio Claro, Lençóis Paulista, SP, Brasil. Canal6, Bauru.

MAFFEI, F., UBAID, F.K. \& JIM, J. 2011b. Anurofauna em área de cerrado aberto no município de Borebi, estado de São Paulo, Sudeste do Brasil: uso do habitat, abundância e variação sazonal. Biota Neotrop. 11(2): http:// www.biotaneotropica.org.br/v11n2/pt/abstract?article+bn04011022011.

MAGURRAN, A.E. 2004. Measuring biological diversity. Blackwell Publishing Company, Oxford.

MELO, A.S. \& FROEHLICH, C.G. 2001. Evaluation of methods for estimating macroinvertebrate species richness using individual stones in tropical streams. Freshwater Biol. 46:711-721. http://dx.doi.org/10.1046/ j.1365-2427.2001.00709.x

MELO, A.S. 2004. A critique of the use of jackknife and related non-parametric techniques to estimate species richness. Community Ecol. 5(2):149-157. http://dx.doi.org/10.1556/ComEc.5.2004.2.1

MITTERMEIER, R.A., GIL, P.R., HOFFMAN, M., PILGRIM, J., BROOKS, T., MITTERMEIER, C.G., LAMOREUX, J. \& FONSECA, G.A.B. 2004. Hotspots revisited: earth's biologically richest and most endangered terrestrial ecoregions. CEMEX \& Agrupacion Sierra Madre, Cidade do México.

MONTEIRO, C.H.B., PRADO, B.H.S. \& DIAS, A.C. 2009. Plano de manejo da Estação Ecológica de Angatuba. Instituto Florestal/SEMA, São Paulo. 
MORELLATO, L.P.C. \& HADDAD, C.F.B. 2000. Introduction: the brazilian Atlantic Forest. Biotropica 32(4b):786-792.

OLIVEIRA, R.J. 2006. Variação da composição florística e da diversidade alfa das florestas atlânticas no estado de São Paulo. Tese de Doutorado, Universidade Estadual de Campinas, Campinas.

OLIVEIRA-FILHO, A.T. \& FONTES, M.A.L. 2000. Patterns of floristic differentiation among atlantic forests in Southeastern Brazil and the influence of climate. Biotropica 32(4b):793-810. http://dx.doi. org/10.1111/j.1744-7429.2000.tb00618.x

OLIVEIRA-FILHO, A.T. \& RATTER, J.A. 2002. Vegetation physiognomies and woody flora of the Cerrado biome. In The cerrados of Brazil: ecology and natural history of a Neotropical Savanna (P.S. Oliveira \& R.J. Marquis, orgs.). Columbia University Press, NewYork, p.91-120.

PEEL, M.C., FINLAYSON, B.L. \& McMAHON, T.A. 2007. Updated world map of the Köppen-Geiger climate classification. Hydrol. Earth Syst. Sci. 11:1633-1644. http://dx.doi.org/10.5194/hess-11-1633-2007

PRADO, D.E. 2000. Seasonally dry forests of Tropical South America: from forgotten ecosystems to a new phytogeographic unit. Edinburgh J. Bot. 57(3):437-461. http://dx.doi.org/10.1017/S096042860000041X

PYRON, R.A. \& WIENS, J.J. 2011. A large-scale phylogeny of Amphibia including over 2800 species, and a revised classification of extant frogs, salamanders, and caecilians. Mol. Phylogenet. Evol. 61(2):543-583. http:// dx.doi.org/10.1016/j.ympev.2011.06.012

RATTER, J.A., RIBEIRO, J.F. \& BRIDGEWATER, S. 1997. The Brazilian Cerrado vegetation and threats to its biodiversity. Ann. Bot. 80:223-230. http://dx.doi.org/10.1006/anbo.1997.0469

RIBEIRO, J.F. \& WALTER, B.M.T. 2008. As principais fitofisionomias do Bioma Cerrado. In Cerrado: ecologia e flora (S.M. Sano, S.P. Almeida \& J.F. Ribeiro, eds.). EMBRAPA, Planaltina, p.153-212.

RIBEIRO, M.C., METZGER, J.P., MARTENSEN, A.C., PONZONI, F.J. \& HIROTA, M.M. 2009. The Brazilian Atlantic Forest: how much is left, and how is the remaining forest distributed? Implications for conservation. Biol. Conserv. 142:1144-1156. http://dx.doi.org/10.1016/j. biocon.2009.02.021

RIBEIRO-JÚNIOR, J.W. \& BERTOLUCI, J. 2009. Anuros do cerrado da Estação Ecológica e da Floresta Estadual de Assis, sudeste do Brasil. Biota Neotrop. 9(1): http://www.biotaneotropica.org.br/v9n1/pt/abstrac t?inventory+bn02709012009 (último acesso em 16/07/2010).

ROSSA-FERES, D.C., MARTINS, M., MARQUES, O.A.V., MARTINS, I.A., SAWAYA, R.J. \& HADDAD, C.F.B. 2008. Herpetofauna. In Diretrizes para a conservação e restauração da biodiversidade no estado de São Paulo (R.R. Rodrigues \& V.LR. Bononi, orgs.). Instituto de Botânica/ SEMA, São Paulo, p.82-94.

ROSSA-FERES, D.C., SAWAYA, R.J., FAIVOVICH, J., GIOVANELLI, J.G.R., BRASILEIRO, C.A., SCHIESARI, L., ALEXANDRINO, J. \& HADDAD, C.F.B. 2011. Anfíbios do Estado de São Paulo, Brasil: Conhecimento Atual e Perspectivas. Biota Neotrop. 11(1a): http://www. biotaneotropica.org.br/v11n1a/pt/abstract?inventory+bn0041101a2011 (último acesso em 22/12/2010).

SANO, E.E., ROSA, R., BRITO, J.L.S. \& FERREIRA, L.G. 2010. Land cover mapping of the tropical savanna region in Brazil. Environ. Monit. Assess. 166:113-124. http://dx.doi.org/10.1007/s10661-009-0988-4

SANTOS, T.G., VASCONCELOS, T.S., ROSSA-FERES, D.C. \& HADDAD, C.F.B. 2009. Anurans of a seasonally dry tropical forest: Morro do Diabo State Park, São Paulo state, Brazil. J. Nat. Hist. 43(15-16):973-993. http:// dx.doi.org/10.1080/00222930802702498

SCOTT JUNIOR, N.J. \& WOODWARD, B.D. 1994. Surveys at breeding sites. In Measuring and monitoring biological diversity: standard methods for amphibians (W.R. Heyer, M.A. Donnelly, R.W. Mc Diarmid, L.C. Hayek \& M.S Foster, eds.). Smithsonian Institution Press, Washington and London, p.118-125.
SEGALLA, M.V., CARAMASCHI, U., CRUZ, C.A.G., GARCIA, P.C.A., GRANT, T., HADDAD, C.F.B \& LANGONE, J. 2012. Brazilian amphibians - List of species. http://www.sbherpetologia.org.br (último acesso em 12/09/2012).

SILVA, J.M.C. \& BATES, J.M. 2002. Biogeographic patterns and conservation in the South American Cerrado: a tropical savanna hotspot. BioScience 52(3):225-233. http://dx.doi.org/10.1641/00063568(2002)052[0225:BPACIT]2.0.CO;2

SILVANO, D. 2011. Distribuição e conservação de anfíbios no Cerrado em cenários atuais e futuros. Tese de Doutorado, Universidade de Brasília, Brasília.

SILVEIRA, L.F., BEISIEGEL, B.M., CURCIO, F.F., VALDUJO, P.H., DIXO, M., VERDADE, V.K., MATTOX, G.M.T. \& CUNNINGHAM, P.T.M. 2010. Para que servem os inventários de fauna? Estudos avançados 68:173-207. http://dx.doi.org/10.1590/S010340142010000100015

TOLEDO, L.F., GIOVANELLI, J.G.R., GIASSON, L.O.M., PRADO, C.P.A., GUIMARÃES, L.D., BASTOS, R.P. \& HADDAD, C.F.B. 2007. Guia interativo dos anfíbios anuros do Cerrado, Campos Rupestres e Pantanal. Neotrópica, São Paulo. CD-ROM.

UETANABARO, M., PRADO, C.P.A., RODRIGUES, D.J., GORDO, M. \& CAMPOS, Z. 2008. Guia de campo dos anuros do Pantanal e planaltos de entorno. UFMS, Campo Grande/UFMT, Cuiabá.

VALDUJO, P.H. 2011. Diversidade e distribuição de anfíbios no Cerrado: o papel dos fatores históricos e dos gradientes ambientais. Tese de Doutorado, Universidade de São Paulo, São Paulo.

VALDUJO, P.H., CAMACHO, A., RECODER, R.S., TEIXEIRA JUNIOR, M., GHELLERE, J.M.B., MOTT, T., NUNES, P.M.S., NOGUEIRA, C. \& RODRIGUES, M.T. 2011. Anfíbios da Estação Ecológica Serra Geral do Tocantins, região do Jalapão, Estados do Tocantins e Bahia. Biota Neotrop. 11(1): http://www.biotaneotropica.org.br/v11n1/pt/ abstract?article+bn03511012011 (último acesso em 22/07/2012).

VALDUJO, P.H., SILVANO, D.L., COLLI, G. \& MARTINS, M. 2012. Anuran species composition and distribution patterns in Brazilian Cerrado, a neotropical hotspot. S. A. J. H. 7(2):63-78. http://dx.doi. org/10.2994/057.007.0209

VASCONCELOS, T.S. \& ROSSA-FERES, D.C. 2005. Diversidade, distribuição espacial e temporal de anfíbios anuros (Amphibia, Anura) na região noroeste do estado de São Paulo. Biota Neotrop. 5(2): http:// www.biotaneotropica.org.br/v5n2/pt/abstract?article+BN01705022005 (último acesso em 15/07/2012).

VASCONCELOS, T.S., SANTOS, T.G., ROSSA-FERES, D.C. \& HADDAD, C.F.B. 2010. Similarity of ground-dwelling anuran (Amphibia) composition among different vegetation physiognomies in a Mesophytic Semideciduous Forest from southeastern Brazil. North-West. J. Zool. 6(2):275-285.

VERDADE, V.K., VALDUJO, P.H., CARNAVAL, A.C., SCHIESARI, L., TOLEDO, L.F., MOTT, T., ANDRADE, G.V., ETEROVICK, P.C., MENIN, M., PIMENTA, B.V.S., NOGUEIRA, C., LISBOA, C.S., PAULA, C.D. \& SILVANO, D.L. 2012. A leap further: the Brazilian Amphibian Conservation Action Plan. Alytes 29(1-4):28-43.

WALTHER, B.A. \& MOORE, J.L. 2005. The concepts of bias, precision and accuracy, and their use in testing the performance of species richness estimators, with a literature review of estimator performance. Ecography 28(6):815-829. http://dx.doi.org/10.1111/j.2005.09067590.04112.x

WERNECK, F.P. \& COLLI, G.R. 2006. The lizard assemblage from Seasonally Dry Tropical Forest enclaves in the Cerrado biome, Brazil, and its association with the Pleistocenic Arc. J. Biogeogr. 33:1983-1992. http://dx.doi.org/10.1111/j.1365-2699.2006.01553.x

WERNECK, F.P. COSTA, G.C., COLLI, G.R., PRADO, D.E. \& SITES JUNIOR, J.W. 2011. Revisiting the historical distribution of Seasonally Dry Tropical Forests: new insights based on palaeodistribution modelling and palynological evidence. Global Ecol. Biogeogr. 20:272-288. http:// dx.doi.org/10.1111/j.1466-8238.2010.00596.x 


\section{Apêndice}

Apêndice 1. Linhas de armadilhas de interceptação e queda e ambientes aquáticos amostrados na Estação Ecológica de Angatuba, SP. Fitofisionomias: AA=área alterada; $\mathrm{CD}=$ cerrado denso; $\mathrm{CE}=$ cerradão; $\mathrm{FES}$ = floresta estacional semidecidual; FESA = floresta estacional semidecidual aluvial.

Appendix 1. Pitfall trap lines and aquatic sites sampled at the Estação Ecológica de Angatuba, São Paulo state, Brazil. Phytophysiognomies: AA = disturbed area; $\mathrm{CD}$ = cerrado denso; CE = cerradão; FES = seasonal semideciduous forest; FESA = seasonal semideciduous alluvial forest.

\begin{tabular}{|c|c|c|}
\hline Linha de armadilha/ambiente aquático & Fitofisionomia & Coordenada geográfica (SAD 69) \\
\hline I & FES & $23^{\circ} 24^{\prime} 48^{\prime \prime} \mathrm{S} / 48^{\circ} 22^{\prime} 31^{\prime \prime} \mathrm{O}$ \\
\hline II & FES & $23^{\circ} 24^{\prime} 40^{\prime \prime} \mathrm{S} / 48^{\circ} 22^{\prime} 52^{\prime \prime} \mathrm{O}$ \\
\hline III & $\mathrm{CE}$ & $23^{\circ} 24^{\prime} 04^{\prime \prime} \mathrm{S} / 48^{\circ} 22^{\prime} 57^{\prime \prime} \mathrm{O}$ \\
\hline IV & CD & $23^{\circ} 23^{\prime} 59^{\prime \prime} \mathrm{S} / 48^{\circ} 22^{\prime} 38^{\prime \prime} \mathrm{O}$ \\
\hline $\mathrm{V}$ & CD & $23^{\circ} 23^{\prime} 49^{\prime \prime} \mathrm{S} / 48^{\circ} 22^{\prime} 25^{\prime \prime} \mathrm{O}$ \\
\hline VI & CD & $23^{\circ} 23^{\prime} 48^{\prime \prime} \mathrm{S} / 48^{\circ} 22^{\prime} 07^{\prime \prime} \mathrm{O}$ \\
\hline VII & $\mathrm{CE}$ & $23^{\circ} 24^{\prime} 22^{\prime \prime} \mathrm{S} / 48^{\circ} 20^{\prime} 45^{\prime \prime} \mathrm{O}$ \\
\hline VIII & $\mathrm{CE}$ & $23^{\circ} 24^{\prime} 33^{\prime \prime} \mathrm{S} / 48^{\circ} 20^{\prime} 58^{\prime \prime} \mathrm{O}$ \\
\hline IX & FES & $23^{\circ} 23^{\prime} 52^{\prime \prime} \mathrm{S} / 48^{\circ} 19^{\prime} 49^{\prime \prime} \mathrm{O}$ \\
\hline $\mathrm{X}$ & FES & $23^{\circ} 24^{\prime} 01^{\prime \prime} \mathrm{S} / 48^{\circ} 19^{\prime} 32^{\prime \prime} \mathrm{O}$ \\
\hline XI & FES & $23^{\circ} 25^{\prime} 14^{\prime \prime} \mathrm{S} / 48^{\circ} 20^{\prime} 08^{\prime \prime} \mathrm{O}$ \\
\hline XII & FES/FESA & $23^{\circ} 25^{\prime} 41^{\prime \prime} \mathrm{S} / 48^{\circ} 19^{\prime} 49^{\prime \prime} \mathrm{O}$ \\
\hline 1 - Lagoa do talhão de Pinus sp. & AA/FES & $23^{\circ} 24^{\prime} 54^{\prime \prime} \mathrm{S} / 48^{\circ} 22^{\prime} 05^{\prime \prime} \mathrm{O}$ \\
\hline 2 - Cachoeira do Sargento & FES & $23^{\circ} 23^{\prime} 53^{\prime \prime} \mathrm{S} / 48^{\circ} 22^{\prime} 37^{\prime \prime} \mathrm{O}$ \\
\hline 3 - Córrego do Sargento & FES & $23^{\circ} 23^{\prime} 46^{\prime \prime} \mathrm{S} / 48^{\circ} 22^{\prime} 00^{\prime \prime} \mathrm{O}$ \\
\hline 4 - Cachoeira das Antas & FES & $23^{\circ} 23^{\prime} 35^{\prime \prime} \mathrm{S} / 48^{\circ} 21^{\prime} 47^{\prime \prime} \mathrm{O}$ \\
\hline 5 - Afluente do córrego da Conquista & FES & $23^{\circ} 24^{\prime} 48^{\prime \prime} \mathrm{S} / 48^{\circ} 21^{\prime} 32^{\prime \prime} \mathrm{O}$ \\
\hline 6 - Represa & AA/FES & $23^{\circ} 25^{\prime} 19^{\prime \prime} \mathrm{S} / 48^{\circ} 20^{\prime} 06^{\prime \prime} \mathrm{O}$ \\
\hline 7 - Rio Guareí & FES/FESA & $23^{\circ} 26^{\prime} 04^{\prime \prime} \mathrm{S} / 48^{\circ} 19^{\prime} 56^{\prime \prime} \mathrm{O}$ \\
\hline 8 - Lagoa do rio Guareí & FESA & $23^{\circ} 26^{\prime} 04^{\prime \prime} \mathrm{S} / 48^{\circ} 19^{\prime} 54^{\prime \prime} \mathrm{O}$ \\
\hline 9 - Córrego Água Bonita & FES/FESA & $23^{\circ} 24^{\prime} 41^{\prime \prime} \mathrm{S} / 48^{\circ} 19^{\prime} 30^{\prime \prime} \mathrm{O}$ \\
\hline 10 - Córrego Água Bonita & FESA & $23^{\circ} 25^{\prime} 11^{\prime \prime} \mathrm{S} / 48^{\circ} 19^{\prime} 29^{\prime \prime} \mathrm{O}$ \\
\hline 11 - Afluente do córrego Água Bonita & FESA & $23^{\circ} 25^{\prime} 13^{\prime \prime} \mathrm{S} / 48^{\circ} 19^{\prime} 16^{\prime \prime} \mathrm{O}$ \\
\hline 12 - Várzea do rio Guareí & FESA & $23^{\circ} 25^{\prime} 16^{\prime \prime} \mathrm{S} / 48^{\circ} 19^{\prime} 11^{\prime \prime} \mathrm{O}$ \\
\hline 13 - Brejo e mata ciliar no entorno & AA/FES & $23^{\circ} 25^{\prime} 33^{\prime \prime} \mathrm{S} / 48^{\circ} 21^{\prime} 09^{\prime \prime} \mathrm{O}$ \\
\hline 14 - Brejo em pastagem & AA & $23^{\circ} 25^{\prime} 40^{\prime \prime} \mathrm{S} / 48^{\circ} 21^{\prime} 26^{\prime \prime} \mathrm{O}$ \\
\hline
\end{tabular}

Apêndice 2. Anuros coletados na Estação Ecológica de Angatuba, SP e depositados na Coleção Herpetológica do Museu de Zoologia, Universidade Estadual de Campinas (ZUEC).

Appendix 2. Anuran species collected at the Estação Ecológica de Angatuba, São Paulo state, Brazil and deposited in the Coleção Herpetológica do Museu de Zoologia, Universidade Estadual de Campinas (ZUEC).

Aplastodiscus perviridis (18812-18814); Chiasmocleis albopunctata (18815-18816); Dendropsophus anceps (18817-18822); Dendropsophus jimi (18823-18824); Dendropsophus minutus (18825-18826); Dendropsophus nanus (18827-18831); Dendropsophus sanborni (18832-18834, 18895); Elachistocleis cesarii (18835); Eupemphix nattereri (18836-18840); Haddadus binotatus (18841-18843); Hypsiboas albopunctatus (18844); Hypsiboas faber (18845-18846); Hypsiboas prasinus (18847-18850); Itapotihyla langsdorffii (18851-18855); Leptodactylus fuscus (18856-18857); Leptodactylus latrans (18858); Leptodactylus mystacinus (18859-18862); Leptodactylus podicipinus (18863); Leptodactylus sp. (gr. marmoratus) (18864-18865); Odontophrynus americanus (18866); Phyllomedusa tetraploidea (18867-18869); Physalaemus cuvieri (18870-18875); Physalaemus olfersii (18876-18877); Rhinella ornata (18878-18882); Rhinella schneideri (20369); Scinax fuscomarginatus (18883-18887); Scinax fuscovarius (18888-18889); Scinax rizibilis (19201-19202); Scinax squalirostris (18890); Sphaenorhynchus caramaschii (18891-18892, 19197-19200); Vitreorana uranoscopa (18893-18894). 